\title{
THE LIMITING ABSORPTION PRINCIPLE FOR THE ACOUSTIC WAVE OPERATORS IN TWO UNBOUNDED MEDIA
}

Dedicated to Professor Mutsuhide Matsumura on his sixtieth birthday

$\mathrm{By}$

\section{Mitsuteru KADOWAKI}

\section{Introduction.}

In the present paper we study the limiting absorption principle for the acoustic wave operators in two unbounded media. We assume that the propagation speed is discontinuous at the interface and the equilibrium density is 1 .

Let $n \geqq 2$ and $x=(y, z) \in \boldsymbol{R}^{n-1} \times \boldsymbol{R}$. The following equation describes the wave propagation here:

$$
\partial_{t}^{2} u(t, x)-a(x)^{2} \Delta u(t, x)=0, \quad(t, x) \in \boldsymbol{R} \times \boldsymbol{R}^{n},
$$

where $a(x)$ is a propagation speed.

We make the assumptions for the interface separating two media and $a(x)$.

Let $\varphi_{0}(y)=a|y|$ and $\varphi(y) \in C^{1}\left(\boldsymbol{R}^{n-1} \backslash\{0\}\right)$, where $a \geqq 0$. We assume that $\varphi(y)$ describes the interface and satisfies

$$
\sum_{|\alpha| \leqq 1}|y|^{|\alpha|}\left|\partial^{\alpha}\left(\varphi(y)-\varphi_{0}(y)\right)\right|=O\left(|y|^{-\theta}\right)(|y| \longrightarrow \infty),
$$

for some $\theta>0$, and

$$
\sum_{|\alpha| \leq 1}|y|^{|\alpha|}\left|\partial^{\alpha} \varphi(y)\right|=O\left(|y|^{-\sigma}\right)(|y| \longrightarrow 0) .
$$

where $0<\sigma<1 / 2$. For $\varphi(y)$, we use the following notation:

$$
\begin{gathered}
\Omega_{+}=\{x=(y, z): z>\varphi(y)\}, \\
\Omega_{-}=\{x=(y, z): z<\varphi(y)\}, \\
S=\{x=(y, z): z=\varphi(y)\} .
\end{gathered}
$$

We denote the unit normal vector at the point $x \in S$ by $\nu=\left(\nu_{1}, \nu_{2}, \cdots, \nu_{z}\right)$ with $\nu_{z}>0$.

The propagation speed $a(x)>0$ is assumed to satisfy the following: for Received January 4, 1992, Revised October 28, 1992. 
some $c>1$,

$$
1 / c<a(x)<c
$$

and there exist $a_{ \pm}>0, a_{\bar{L}}^{ \pm}(x) \in \boldsymbol{B}^{1}\left(\Omega_{ \pm}\right)$and $a_{S}(x) \in L^{\infty}\left(\boldsymbol{R}^{n}\right)$ such that $a(x)$ is decomposed as

$$
\left\{\begin{array}{l}
a(x)=a_{ \pm}+a_{L}^{ \pm}(x)+a_{S}(x)\left(x \in \Omega_{ \pm}\right), \\
\sum_{|\alpha| \leq 1}|x|^{|\alpha|}\left|\partial^{\alpha} a_{L}^{ \pm}(x)\right|=O\left(|x|^{-\theta}\right)\left(|x| \longrightarrow \infty, x \in \Omega_{ \pm}\right), \\
a_{S}(x)=O\left(|x|^{-\theta-1}\right)(|x| \longrightarrow \infty)
\end{array}\right.
$$

for some $\theta>0$.

Under (A.0) (A.3), we show the nonexistence of eigenvalues and the limiting absorption principle of the acoustic operator $-a(x)^{2} \Delta$ for (1.1).

There are many works dealing with the acoustic wave propagation problem with the discontinuous propagation speed at the interface separating media. Eidus [5] proved the limiting absorption and amplitude principle for two unbounded media problem with the interface satisfying the following conditions: for any $x \in S$

$$
\begin{aligned}
& \nu_{2} \geqq C_{1}>0, \\
& |x \cdot \nu| \leqq C_{2},
\end{aligned}
$$

where $C_{j}>0,(j=1,2)$, are independent of $x \in S$. For example,

$$
\varphi(y) \in C^{1}\left(\boldsymbol{R}^{n-1}\right), \quad \varphi(y)=\frac{\sin |y|}{|y|}(|y| \gg 1), \quad \varphi_{0}(y)=0
$$

satisfies (1.2) and (1.3), but not satisfies (A.0). We can also deal with the following interface not satisfying (1.2),

where, $0<\sigma<1 / 2$.

$$
\varphi(y)=|y|^{-\sigma}, \quad \varphi_{0}(y)=0
$$

The propagation speed considered in Eidus [5] is a piecewise constant function while we can perturb the propagation speed. Wilcox [17] considered two stratified fluilds in a half space and established the eigenfunction expansion theorem. Ben-Artzi [1], Weder [14] [16] and Dermenjian and Guillot [3] considered perturbed stratified fluilds problems. They showed the limiting absorption principle by the approach of Wilcox [17]. Kikuchi and Tamura [8] also proved the limiting amplitude principle for perturbed stratified fluilds. On the other hand there are some works dealing with the case where the equilibrium density is discontinuous at the interface separating media, for example, 
Debièvre and Pravica [2] and Wilcox [17].

In order to show the limiting absorption principle for our operator, we use Mourre's method. This method was first developed by Mourre [9] to prove the limiting absorption principle for 3-body Schrödinger operators (see also Perry, Sigal and Simon [10] and Tamura [12]). In Froese and Herbst [6], they showed by Mourre's method that N-body Schrödinger operators have no positive eigenvalues. Iwashita [7] and Weder [13] showed the limiting absorption principle for first order symmetric systems. For the acoustic wave operators in perturbed stratified fluilds, Debièrve and Pravica [2] obtained the similar results as in [7] and [13]. Tamura [11] used Mourre's method in order to prove the limiting amplitude principle for the acoustic wave operators (see also Kikuchi and Tamura [8]).

We now define the acoustic operator $L$ as

$$
L=-a(x)^{2} \Delta
$$

Under the above assumptions, (A.0) (A.3), $L$ is a symmetric operator in the Hilbert space $L^{2}\left(\boldsymbol{R}^{n} ; E(x) d x\right)$ with $E(x)=a(x)^{-2}$ and admits a unique selfadjoint realization. We denote by the same notation $L$ this self-adjoint realization. Then $L$ is a positive operator (zero is not an eigenvalue) and the domain $D(L)$ is given by $D(L)=H^{2}\left(\boldsymbol{R}_{x}^{n}\right), H^{s}\left(\boldsymbol{R}_{x}^{n}\right)$ being the Sobolev space of order s over $\boldsymbol{R}_{x}^{n}$. We also denote by $R(z ; L)$ the resolvent $(L-z)^{-1}$ of $L$ for $\operatorname{Im} z: \neq 0$.

We need several notations to describe our results. Let $L^{2}$ be the usual $L^{2}$ space defined on $\boldsymbol{R}^{n}$, with the inner product

$$
\langle u, v\rangle=\int u(x) \overline{v(x)} d x
$$

and the corresponding norm $|\cdot|_{0}$. For $\alpha \in \boldsymbol{R}$ let $L_{\alpha}^{2}$ be the weighted $L^{2}$ space defined by

$$
L_{\alpha}^{2}=\left\{u(x):\langle x\rangle^{\alpha} u(x) \in L^{2}\left(\boldsymbol{R}_{x}^{n}\right)\right\},\langle x\rangle=\left(1+|x|^{2}\right)^{1 / 2},
$$

with the norm

$$
|u|_{\alpha}^{2}=\int\langle x\rangle^{2 \alpha}|u(x)|^{2} d x .
$$

Let $A: L^{2} \rightarrow L^{2}$ be a bounded operator. We denote by $\|A\|$ the operator norm of $A$.

The main result is

Theorem 1.1. Assume that (A.0) (A.3). Then

(i) L has no eigenvalues. 
(ii) Let $\lambda_{0}>0$ and $\alpha>1 / 2$. Then for any compact interval $I \subset \boldsymbol{R}_{+}$containing $\lambda_{0}$, there exists a positive constant $C=C(I, \alpha)$ such that

$$
\left\|\langle x\rangle^{-\alpha} R(\lambda \pm i \kappa ; L)\langle x\rangle^{-\alpha}\right\| \leqq C,
$$

for $\lambda \in I, 0<\kappa<1$.

(iii) For every $\lambda>0$ and $\alpha>1 / 2$, following two limits

$$
R(\lambda \pm i 0 ; L)=\lim _{\kappa \downarrow 0} R(\lambda \pm i \kappa ; L),
$$

exist in the uniform operator topology of $\mathfrak{B}\left(L_{\alpha}^{2}, L_{-\alpha}^{2}\right)$. Moreover $R(\lambda \pm i 0 ; L)$ are locally Hölder continuous.

Acknowledgement. The author would like to express his sincere gratitute to Professors M. Matsumura and K. Mochizuki for their generous advice and kind encouragement.

\section{Mourre's estimate.}

In this section we shall show Mourre's estimate (Lemma 2.4). First, we prove the following lemma. We need this lemma to estimate an integral on the interface $S$.

Lemma 2.2. Let $s>1 / 2$. For $u \in \boldsymbol{S}\left(\boldsymbol{R}^{n}\right)$ (Schwartz space), we define

$$
\left(T_{\varphi} u\right)(y)=u(y, \varphi(y)) .
$$

$T_{\varphi}$ has an extension to a bounded operator from $H^{3}\left(\boldsymbol{R}^{n}\right)$ to $L^{2}\left(\boldsymbol{R}^{n-1}\right)$.

Proof. We show that:

$$
\left|T_{\varphi} u\right|_{L 2\left(R^{n-1}\right)} \leqq C|u|_{H^{s\left(R^{n}\right)}}
$$

for $u \in \boldsymbol{S}\left(\boldsymbol{R}^{n}\right)$. Let $u \in \boldsymbol{S}\left(\boldsymbol{R}^{n}\right)$. Then $u(y, \varphi(y))$ is represented as

$$
u(y, \varphi(y))=(2 \pi)^{-1 / 2} \int_{-\infty}^{+\infty} e^{i \varphi(y) \cdot \zeta}\left(\mathfrak{F}_{z} u\right)(y, \zeta) d \zeta,
$$

where $\boldsymbol{\xi}=(\eta, \zeta) \in \boldsymbol{R}_{\eta}^{n-1} \times \boldsymbol{R}_{\zeta}=\boldsymbol{R}_{\xi}^{n}$ and $\mathfrak{F}_{z}$ is the Fourier transform in $\boldsymbol{R}_{\boldsymbol{z}}$. By Schwartz's inequality, we have

$$
\left|\left(T_{\varphi} u\right)(y)\right|^{2} \leqq(2 \pi)^{-1} \int_{-\infty}^{+\infty}\left(1+\zeta^{2}\right)^{-8} d \zeta \int_{-\infty}^{+\infty}\left|\left(1+\zeta^{2}\right)^{s / 2}\left(\mathfrak{\mho}_{z} u\right)(y, \zeta)\right|^{2} d \zeta
$$

By integrating both sides of the above inequality over $\boldsymbol{R}^{n-1}$, we obtain $(2.1)$.

We consider only the case $1=a_{-}^{-2}<a_{+}^{-2}$. The other cases can be proved similarly. 
We define the self-adjoint operator $H(\lambda)$ on $L^{2}$ by

Then we have

$$
\begin{aligned}
& H(\lambda)=-\Delta-\lambda(E(x)-1), \\
& D(H(\lambda))=H^{2}\left(\boldsymbol{R}^{n}\right)
\end{aligned}
$$

$$
(L-(\lambda \pm i \kappa))^{-1}=(H(\lambda)-\lambda \mp i \kappa E(x))^{-1} E(x) .
$$

We apply Mourre's commutator method to $H(\lambda)$ on $L^{2}$ (see Tamura [11] or Kikuchi and Tamura [8]).

By (A.3), we can decompose $E(x)=E_{L}^{ \pm}(x)+E_{S}(x)\left(x \in \Omega_{ \pm}\right)$in such a way that

$$
\begin{aligned}
& \sum_{|\alpha| \leqq 1}|x|^{|\alpha|}\left|\partial^{\alpha}\left(E_{\bar{L}}^{ \pm}(x)-a_{ \pm}^{-2}\right)\right|=O\left(|x|^{-\theta}\right),\left(|x| \longrightarrow \infty, x \in \Omega_{ \pm}\right), \\
& E_{S}(x)=O\left(|x|^{-1-\theta}\right),(|x| \longrightarrow \infty) .
\end{aligned}
$$

Let $A$ be the generator of the dilation unitary grup;

$$
A=\frac{1}{2 i}(x \cdot \nabla+\nabla \cdot x) \text {. }
$$

We define the commutator $i[H(\lambda), A]$ as a form on $H^{2}\left(\boldsymbol{R}^{n}\right) \cap D(A)$ as follows; For $u, v \in H^{2}\left(\boldsymbol{R}^{n}\right) \cap D(A)$

$$
\begin{aligned}
& \langle i[H(\lambda), A] u, v\rangle \\
& =i(\langle A u, H(\lambda) v\rangle-\langle H(\lambda) u, A v\rangle) .
\end{aligned}
$$

LEMMA 2.2. The form $i[H(\lambda), A]$ defined on $H^{2}\left(\boldsymbol{R}^{n}\right) \cap D(A)$ is extended to a bounded operator from $H^{1}\left(\boldsymbol{R}^{n}\right)$ to $H^{-1}\left(\boldsymbol{R}^{n}\right)$ which is denoted by $i[H(\lambda), A]^{0}$.

In order to prove Lemma 2.2, we need the following lemma.

I EMMA 2.3 (Sobolev's Theorem). Suppose that

$$
1 / 2-l / m=1 / q, 2<q<\infty \text {. }
$$

Then we have the embedding

$$
H^{l}\left(\boldsymbol{R}^{m}\right) \subset L^{q}\left(\boldsymbol{R}^{m}\right) .
$$

Proof of Lemma 2.2. Let $u \in \boldsymbol{S}\left(\boldsymbol{R}^{n}\right)$. Then we have

$$
\begin{aligned}
& \quad\langle i[-\Delta, A] u, u\rangle=2\langle\nabla u, \nabla u\rangle, \\
& \langle i[E-1, A] u, u\rangle \\
& =\left\langle x \cdot \nabla u, E_{L} u\right\rangle+\left\langle E_{L} u, x \cdot \nabla u\right\rangle+n\left\langle E_{L} u, u\right\rangle, \\
& +\left\langle x \cdot \nabla u, E_{S} u\right\rangle+\left\langle E_{S} u, x \cdot \nabla u\right\rangle+n\left\langle E_{S} u, u\right\rangle,
\end{aligned}
$$


where $E_{L}=E_{L}^{ \pm}(x)\left(x \in \Omega_{ \pm}\right)$.

We calculate the first and the second terms of right side of (2.5). We set $w_{n}=z-\varphi(y)$ and $w_{j}=y_{j}(j=1,2, \cdots, n-1)$. Then we have $D(y, z) / D\left(w^{\prime}, w_{n}\right)$ $=1$, where $w^{\prime}=\left(w_{1}, w_{2}, \cdots, w_{n-1}\right)$. Integrating by parts, we have

$$
\begin{aligned}
\langle x \cdot \nabla u, & \left.E_{L}^{ \pm} u\right\rangle_{L^{2}\left(\Omega_{ \pm}\right)}+\left\langle E_{L}^{ \pm} u, x \cdot \nabla u\right\rangle_{L^{2}\left(\Omega_{ \pm}\right)} \\
= & \left\langle w \cdot \nabla_{w} \tilde{u}, \tilde{E}_{L}^{ \pm} \tilde{u}\right\rangle_{L^{2}\left(R_{ \pm}^{n}\right)}+\left\langle\tilde{E}_{L}^{ \pm} \tilde{u}, w \cdot \nabla_{w} \tilde{u}\right\rangle_{L^{2}\left(\boldsymbol{R}_{ \pm}^{n}\right)} \\
& -\left\langle\left(w^{\prime} \cdot \nabla_{w^{\prime}} \varphi\left(w^{\prime}\right)-\varphi\left(w^{\prime}\right)\right) \partial_{w_{n}} \tilde{u}, \tilde{E}_{L}^{ \pm} \tilde{u}\right\rangle_{L^{2}\left(\boldsymbol{R}_{ \pm}^{n}\right)} \\
& -\left\langle\tilde{E}_{\bar{L}}^{ \pm} \tilde{u},\left(w^{\prime} \cdot \nabla_{w^{\prime}} \varphi\left(w^{\prime}\right)-\varphi\left(w^{\prime}\right)\right) \partial_{w_{n}} \tilde{u}\right\rangle_{L^{2}\left(\boldsymbol{R}_{ \pm}^{n}\right)} \\
= & -\left\langle\left(w \cdot \nabla_{w} \tilde{E}_{L}^{ \pm}\right) \tilde{u}, \tilde{u}\right\rangle_{L^{2}\left(\boldsymbol{R}_{ \pm}^{n}\right)}-n\left\langle\tilde{E}_{L}^{ \pm} \tilde{u}, \tilde{u}\right\rangle_{L^{2}\left(\boldsymbol{R}_{ \pm}^{n}\right)} \\
& +\left\langle\left(w^{\prime} \cdot \nabla_{w^{\prime}} \varphi\left(w^{\prime}\right)-\varphi\left(w^{\prime}\right)\right)\left(\partial_{w_{n}} \tilde{E}_{L}^{ \pm}\right) \tilde{u}, \tilde{u}\right\rangle_{L^{2}\left(\boldsymbol{R}_{ \pm}^{n}\right)} \\
& \pm \int_{\boldsymbol{R}^{n-1}}\left(w^{\prime} \cdot \nabla_{w^{\prime}} \varphi\left(w^{\prime}\right)-\varphi\left(w^{\prime}\right)\right) \tilde{E}_{\bar{L}}^{ \pm}\left(w^{\prime}, 0\right)\left|\tilde{u}\left(w^{\prime}, 0\right)\right|^{2} d w^{\prime} \\
= & -\left\langle\left(x \cdot \nabla E_{E}^{ \pm}\right) u, u\right\rangle_{L^{2}\left(\Omega_{ \pm}\right)}-n\left\langle E_{L}^{ \pm} u, u\right\rangle_{L^{2}\left(\Omega_{ \pm}\right)} \\
& \pm \int_{\boldsymbol{R}^{n-1}}\left(y \cdot \nabla_{y} \varphi(y)-\varphi(y)\right) E_{L}^{ \pm}(y, \varphi(y))|u(y, \varphi(y))|^{2} d y,
\end{aligned}
$$

where $\tilde{E}_{L}^{ \pm}=E_{\tilde{L}}^{ \pm}\left(w^{\prime}, w_{n}+\varphi\left(w^{\prime}\right)\right)$ and $\tilde{u}=u\left(w^{\prime}, w_{n}+\varphi\left(w^{\prime}\right)\right)$. (2.6) implies

$$
\begin{aligned}
& \left\langle x \cdot \nabla u, E_{L} u\right\rangle+\left\langle E_{L} u, x \cdot \nabla u\right\rangle+n\left\langle E_{L} u, u\right\rangle \\
& =-\left\langle\left(x \cdot \nabla E_{L}\right) u, u\right\rangle+\int_{R^{n-1}}\left(y \cdot \nabla_{y} \varphi(y)-\varphi(y)\right)\left(E_{L}^{+0}-E_{L}^{-0}\right)|u(y, \varphi(y))|^{2} d y,
\end{aligned}
$$

where $E_{L}^{ \pm 0}=E_{L}^{ \pm}(y, \varphi(y))$ and $\left(x \cdot \nabla E_{L}\right)=x \cdot \nabla E_{L}^{ \pm}\left(x \in \Omega_{ \pm}\right)$. By (2.4), (2.5) and (2.7), we have

$$
\begin{aligned}
& \langle i[H(\lambda), A] u, u\rangle=2\langle\nabla u, \nabla u\rangle+\lambda\left\langle\left(x \cdot \nabla E_{L}\right) u, u\right\rangle \\
& \quad-\lambda \int_{R^{n-1}}\left(y \cdot \nabla_{y} \varphi(y)-\varphi(y)\right)\left(E_{L}^{+0}-E_{L}^{-0}\right)|u(y, \varphi(y))|^{2} d y \\
& -n \lambda\left\langle E_{S} u, u\right\rangle-\lambda\left\langle E_{S} u, x \cdot \nabla u\right\rangle-\lambda\left\langle x \cdot \nabla u, E_{S} u\right\rangle .
\end{aligned}
$$

Ror any $r>0$, we have

$$
\begin{aligned}
\lambda \int_{R^{n-1}} & \left(y \cdot \nabla_{y} \varphi-\varphi\right)\left(E_{L}^{+0}-E_{L}^{-0}\right)|u(y, \varphi(y))|^{2} d y \\
= & \lambda\left\langle T_{\varphi}^{*}\left(y \cdot \nabla_{y} \varphi-\varphi\right)\left(E_{L}^{+0}-E_{L}^{-0}\right) T_{\varphi} u, u\right\rangle \\
= & \lambda\left(\left\langle T_{\varphi}^{*}\left(\chi_{|y|<r}\left(y \cdot \nabla_{y} \varphi-\varphi\right)\left(E_{L}^{+0}-E_{L}^{-0}\right)\right) T_{\varphi} u, u\right\rangle\right. \\
& \left.+\left\langle T_{\varphi}^{*}\left(\chi_{|y|>r}\left(y \cdot \nabla_{y} \varphi-\varphi\right)\left(E_{L}^{+0}-E_{L}^{-0}\right)\right) T_{\varphi} u, u\right\rangle\right)
\end{aligned}
$$

We define an operator $R_{\varphi}^{r}$ as 


$$
R_{\varphi}^{r} u=\chi_{|y|<r}(y)\left(y \cdot \nabla_{y} \varphi(y)-\varphi(y)\right)^{1 / 2} T_{\varphi} u .
$$

We show that $R_{\varphi}^{r}$ is a bounded operator from $H^{1}\left(\boldsymbol{R}^{n}\right)$ to $L^{2}\left(\boldsymbol{R}^{n-1}\right)$.

If $0<\sigma<1 / 2$, there exist some $s>1 / 2$ and $p>n-1$ such that

$$
\begin{aligned}
& n-1-\sigma p>0, \\
& \frac{n-1}{2 p}+s=1 .
\end{aligned}
$$

By (2.2), (2.3) and Hölder inequality, we have

$$
\begin{aligned}
\left|R_{\varphi}^{r} u\right|_{L^{2}\left(R^{n-1}\right)}^{2} & \leqq C \int_{-\infty}^{+\infty}\left(1+\zeta^{2}\right)^{s} \int_{|y|<r}\left|y \cdot \nabla_{y} \varphi-\varphi\right|\left|\left(\mathfrak{F}_{z} u\right)(y, \zeta)\right|^{2} d y d \zeta \\
& \leqq C r^{(n-1-\sigma p) / p} \int_{-\infty}^{+\infty}\left(1+\zeta^{2}\right)^{s}\left|\left(\mathfrak{F}_{z} u\right)(\cdot, \zeta)\right|_{L^{2 p /(p-1)(R n-1)}}^{2} d \zeta,
\end{aligned}
$$

where $C>0$ is independent of $r$.

Lemma 2.3 implies that

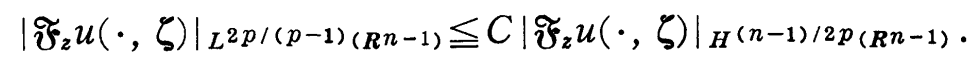

It follows from (2.11), (2.12) and (2.13) that

$$
\left|R_{\varphi}^{r} u\right|_{L^{2(R}\left(R^{n-1)}\right.} \leqq C r^{(n-1-\sigma p) / 2 p}|u|_{H^{1\left(R^{n}\right)}},
$$

where $C$ is independent of $r$.

It follows from Lemma 2.1 that $T_{\varphi}^{*}$ is a bounded operator from $L^{2}\left(\boldsymbol{R}^{n-1}\right)$ to $H^{-1}\left(\boldsymbol{R}^{n}\right)$. Moreover, since $E_{S} x \cdot \nabla$ is a bounded operator from $H^{1}\left(\boldsymbol{R}^{n}\right)$ to $L^{2}\left(\boldsymbol{R}^{n}\right)$, the adjoint operator $(\nabla)^{*} \cdot x E_{S}$ is a bounded operator from $L^{2}\left(\boldsymbol{R}^{n}\right)$ to $H^{-1}\left(\boldsymbol{R}^{n}\right)$.

Hence, from (2.8), (2.9) and (2.13), the form $i[H(\lambda), A]$ on $\boldsymbol{S}\left(\boldsymbol{R}^{n}\right)$ has an extension to a bounded operator $i[H(\lambda), A]^{0}$ on from $H\left(\boldsymbol{R}^{n}\right)$ to $H^{-1}\left(\boldsymbol{R}^{n}\right)$.

Let $u \in H^{2}\left(\boldsymbol{R}^{n}\right) \cap D(A)$. Repeating the argument in the proof of Lemma 2.1 of Weder [13], we can show that $u$ satisfies (2.8).

LEMMA 2.4. Let $\lambda_{0}>0$ and $0<\delta<\min \left(1, \lambda_{0} / 4\right)$ and take $f_{\delta}(p) \in C_{0}^{\infty}(\boldsymbol{R}), 0 \leqq$ $f_{\delta} \leqq 1$ such that $f_{\delta}$ has support in $\left(\lambda_{0}-3 \delta, \lambda_{0}+3 \delta\right)$ and $f_{\delta}=1$ on $\left[\lambda_{0}-2 \delta, \lambda+2 \delta\right]$. Then, there exist a positive constant $\alpha$ and a compact operator $K$ on $L^{2}$ which depend on only $\lambda_{0}$ such that

$$
\begin{aligned}
& f_{\delta}(H(\lambda)) i[H(\lambda), A]^{0} f_{\delta}(H(\lambda)) \\
& \quad \geqq \alpha f_{\delta}(H(\lambda))^{2}+f_{\delta}(H(\lambda)) K(\lambda) f_{\delta}(H(\lambda))
\end{aligned}
$$

for $\lambda \in\left(\lambda_{0}-\delta, \lambda_{0}+\delta\right)$, in the form sence. 
Proof. We simply write $f_{\delta}$ instead of $f_{\delta}(H(\lambda))$. By Lemma 2.2, we have for $u \in L^{2}$,

$$
\begin{aligned}
\left\langle f_{\delta} i[\right. & \left.\left.H(\lambda), A^{0}\right]^{0} f_{\delta} u, u\right\rangle \\
= & 2\left\langle-\Delta f_{\delta} u, f_{\delta} u\right\rangle+\lambda\left(\left\langle\left(\left(x \cdot \nabla E_{L}\right)-n E_{S}\right) f_{\delta} u, f_{\delta} u\right\rangle\right. \\
& -\left\langle x \cdot \nabla f_{\delta} u, E_{S} f_{\delta} u\right\rangle-\left\langle E_{S} f_{\delta} u, x \cdot \nabla f_{\delta} u\right\rangle \\
& -\left\langle\left(T_{\varphi}\right) * \chi_{|y|>r}\left(y \cdot \nabla_{y} \varphi-\varphi\right)\left(E_{L}^{+0}-E_{L}^{-0}\right) T_{\varphi} f_{\delta} u, f_{\delta} u\right\rangle \\
& \left.-\left\langle\left(R_{\varphi}^{r}\right)^{*}\left(E_{L}^{+}-E_{L}^{-0}\right) R_{\varphi}^{r} f_{\delta} u, f_{\delta} u\right\rangle\right) .
\end{aligned}
$$

Let $0<r \ll 1$. By (2.13), there exists a positive number $C$ independent of $r$ such that,

$$
\begin{aligned}
\mid \lambda\langle & \left.\left(R_{\varphi}^{r}\right) *\left(E_{L}^{+0}-E_{L}^{-0}\right) R_{\varphi}^{r} f_{\delta} u, f_{\delta} u\right\rangle \mid \\
& =\left|\lambda\left\langle\left(E_{L}^{+0}-E_{L}^{-0}\right) R_{\varphi}^{r} f_{\delta} u, R_{\varphi}^{r} f_{\delta} u\right\rangle_{L^{2}\left(R^{n-1}\right)}\right| \\
& \leqq C r^{(n-1-\sigma p) / p}\left(\left|\nabla f_{\delta} u\right|_{0}^{2}+\left|f_{\delta}\right|_{0}^{2}\right) .
\end{aligned}
$$

Let $E_{0}(x)=a_{ \pm}^{-2}\left(x \in \Omega_{ \pm}\right)$. Then we have

$$
\begin{aligned}
2\langle-\Delta & \left.f_{\delta} u, f_{\delta} u\right\rangle-\lambda\left\langle\left(R_{\varphi}^{r}\right) *\left(E_{L}^{+0}-E_{L}^{-0}\right) R_{\varphi}^{r} f_{\delta} u, f_{\delta} u\right\rangle \\
\geqq & \left(2-C r^{(n-1-\sigma p) / p}\right)\left\langle H(\lambda) f_{\delta} u, f_{\delta} u\right\rangle-C r^{(n-1-\sigma p) / p}\left|f_{\varepsilon} u\right|_{0}^{2} \\
& +\left(2-C r^{(n-1-\sigma p) / p}\right) \lambda\left\langle\left(E-E_{0}\right) f_{\delta} u, f_{\delta} u\right\rangle \\
& +\left(2-C r^{(n-1-\sigma p) / p}\right) \lambda\left\langle\left(E_{0}-1\right) f_{\delta} u, f_{\delta} u\right\rangle \\
\geqq & \left(\frac{\left(2-C r^{(n-1-\sigma p) / p}\right) \lambda_{0}}{4} C r^{(n-1-\sigma p) / p}\right)\left|f_{\delta} u\right|_{0}^{2} \\
& +\left(2-C r^{(n-1-\sigma p) / p}\right) \lambda\left\langle\left(E-E_{0}\right) f_{\delta} u, f_{\delta} u\right\rangle .
\end{aligned}
$$

Take $h \in C_{0}^{\infty}(\boldsymbol{R}), 0 \leqq h \leqq 1$ such that $h=1$ on $\left(\lambda_{0} / 4,7 \lambda_{0} / 4\right)$. Using $h$, we define an operator $K(\lambda)$ as

$$
K(\lambda)=\sum_{j=1}^{4} K_{j}(\lambda)
$$

where,

$$
\begin{aligned}
& K_{1}(\lambda)=\lambda\left(h(H(\lambda))\left(\left(x \cdot \nabla E_{L}\right)-n E_{S}+\left(2-C r^{(n-1-\sigma p) / p}\right)\left(E-E_{0}\right)\right) h(H(\lambda)),\right. \\
& K_{2}(\lambda)=-\lambda h(H(\lambda)) E_{S} x \cdot \nabla h(H(\lambda)), \\
& K_{3}(\lambda)=-\lambda h(H(\lambda)) \nabla^{*} \cdot x E_{S} h(H(\lambda)), \\
& K_{4}(\lambda)=-\lambda h(H(\lambda)) T_{\varphi}^{*}\left(E_{L}^{+0}-E_{L}^{-0}\right) \chi_{|y|>r}(y \cdot \nabla \varphi-\varphi) T_{\varphi} h(H(\lambda)) .
\end{aligned}
$$

For each $\lambda \in\left(\lambda_{0}-\delta, \lambda_{0}+\delta\right)$, we show that $K_{j}(\lambda)(j=1,2,3,4)$ is a compact 
operator on $L^{2}$.

Noting that $h(H(\lambda))$ is a bounded operator from $L^{2}\left(\boldsymbol{R}^{n}\right)$ to $H^{2}\left(\boldsymbol{R}^{n}\right)$, by Rellich's theorem and (A.3), we have that $K_{j}(\lambda)(j=1,2,3)$ is a compact operator on $L^{2}$. Let $u \in L^{2}$. Noting that

$$
\nabla_{y} T_{\varphi} h(H(\lambda)) u=T_{\varphi} \nabla_{y} h(H(\lambda)) u+\left(\nabla_{y} \varphi(y)\right) T_{\varphi} \partial_{z} h(H(\lambda)) u,
$$

we have that $T_{\varphi} h(H(\lambda))$ is a bounded operator from $L^{2}\left(\boldsymbol{R}^{n}\right)$ to $H^{1}(|y|>r)$. Thus, Rellich's theorem and (A.0) imply that $K_{4}(\lambda)$ is also a compact on $L^{2}$. Thus we obtain (2.15) by (2.16) and (2.17).

Remark. Assuming that $0<\sigma<1$, we can also prove that Lemma 2.2 and 2.3. But, we need the condition $0<\sigma<1 / 2$ to prove the limiting absorption principle (see Appendix).

\section{The limiting absorption principle.}

Sketch OF THE PROOF OF 'THEOREM 1.1 (I ): Assume that $u \in H^{2}\left(\boldsymbol{R}^{n}\right)$ is the eigenfunction associated with eigenvalue $\lambda_{0}>0$ i. e. $L u=\lambda_{0} u$, then we can consider $u$ as the eigenfuncton of $H\left(\lambda_{0}\right)$ associated with eigenvalue $\lambda_{0}$. Using Lemma 2.1 and Lemma 2.2, we can prove

$$
\exp (\alpha\langle x\rangle) u \in L^{2}(\forall \alpha \geqq 0)
$$

in the same way as in Froese and Herbst [6]. Moreover, we can also prove that $u=0$ in the same way as in Appendix I of Tamura [11].

If $|\mu|>1,(A+i \mu)^{-1}$ sends $H^{k}\left(\boldsymbol{R}^{n}\right)$ into $H^{k}\left(\boldsymbol{R}^{n}\right)$ and as an operator on $H^{k}\left(\boldsymbol{R}^{n}\right)(k=0, \pm 1, \pm 2)$

$$
s-\lim _{|\mu| \rightarrow \infty} i \mu(A+i \mu)^{-1}=I
$$

is valid (see Lemma 2.3 of Weder [13]). Put $A(\mu)=i \mu A(A+i \mu)^{-1}$. It follows from $A(\mu)=i \mu+\mu^{2}(A+i \mu)^{-1}$ that $H(\lambda) A(\mu)-A(\mu) H(\lambda)$ is a bounded operator from $H^{1}\left(\boldsymbol{R}^{n}\right)$ to $H^{-1}\left(\boldsymbol{R}^{n}\right)$. Using (3.1), we can prove the following lemma in the same way as the proof of Lemma 2.4 of Weder [13].

LNMMA 3.1 .

$$
i[H(\lambda), A(\mu)]=i \mu(A+i \mu)^{-1} i[H(\lambda), A]^{0} i \mu(A+i \mu)^{-1}
$$

and

$$
\begin{gathered}
s-\lim _{\mu \rightarrow \infty}(-\Delta+1)^{-1 / 2} i[H(\lambda), A(\mu)](-\Delta+1)^{-1 / 2} \\
=(-\Delta+1)^{-1 / 2} i[H(\lambda), A]^{0}(-\Delta+1)^{-1 / 2}
\end{gathered}
$$


for all $\lambda \in\left(\lambda_{0}-\delta, \lambda_{0}+\delta\right)$, as an operator on $L^{2}$.

Using Lemma 3.1, we can also prove the following lemma in the same way as the proof of Lemma 2.5 of Weder [13].

Lemma 3.3. Let $f \in C_{0}^{\infty}\left(\boldsymbol{R}^{n}\right)$. Then

(i ) $f(H(\lambda))$ sends $D(A)$ into $D(A)$

(ii) $[f(H(\lambda)), A]$ defined as operator on $D(A)$ is extended to a bounded operator on $L^{2}$ which is denoted by $[f(H(\lambda)), A]^{0}$.

Following Tamura [12], we consider cut off functions, $\chi_{n}(x) \in C_{0}^{\infty}\left(\boldsymbol{R}^{n}\right)$ such that $\chi_{n}(x)$ has support in $\left\{x \in \boldsymbol{R}^{n} ;|x|<2\right\}$ and $\chi_{n}=1$ for $|x| \leqq 1$. For $\varepsilon>0$ small enough, we define

and

$$
\begin{aligned}
& E_{L, \varepsilon}(x)=E_{0}(x)+\chi_{n}(\varepsilon x)\left(E_{L}(x)-E_{0}(x)\right), \\
& E_{S, \varepsilon}(x)=\chi_{n}(\varepsilon x) E_{S}(x),
\end{aligned}
$$

$$
V_{\varepsilon}^{r}(y)=\chi_{|y|>r}(y) \chi_{n-1}(\varepsilon y)\left(y \cdot \nabla_{y} \varphi(y)-\varphi(y)\right) .
$$

We further define an operator $B(\varepsilon ; \lambda)$ as

$$
\begin{aligned}
B(\varepsilon ; \lambda)= & -2 \Delta+\lambda\left(\left(x \cdot \nabla E_{L, \varepsilon}\right)-n E_{S, \varepsilon}+\nabla^{*} \cdot x E_{S, \varepsilon}-E_{S, \varepsilon} x \cdot \nabla\right. \\
& \left.-T_{\varphi}^{*} V_{\varepsilon}^{r}(y)\left(E_{L}^{+}-E_{L}^{-}\right) T_{\varphi}-\left(R_{\varphi}^{r}\right)^{*}\left(E_{L}^{+}-E_{L}^{-}\right) R_{\varphi}^{r}\right) .
\end{aligned}
$$

Note that by the proof of Lemma 2.2, we can consider $B(\varepsilon ; \lambda)$ to be a bounded operator from $H^{1}\left(\boldsymbol{R}^{n}\right)$ to $H^{-1}\left(\boldsymbol{R}^{n}\right)$.

LEMMA 3.3. Let $M(\varepsilon ; \lambda)=f_{\delta}(H(\lambda)) B(\varepsilon ; \lambda) f_{\delta}(H(\lambda))$. Then $[M(\varepsilon ; \lambda), A]$ defined as a form on $D(A)$ is extended to a bounded operator on $L^{2}$ which is denoted by $[M(\varepsilon ; \lambda), A]^{0}$.

Proof. We again write $f_{\delta}$ for $f_{\delta}(H(\lambda))$. Noting that $M(\varepsilon ; \lambda)$ is a bounded operator on $L^{2}$, we can see that $[M(\varepsilon ; \lambda), A(\mu)]$ is also a bounded operator on $L^{2}$. Let $u \in L^{2}$. Since $A(\mu)$ sends $H^{2}\left(\boldsymbol{R}^{n}\right)$ into $H^{2}\left(\boldsymbol{R}^{n}\right)$, we have

$$
\begin{aligned}
\langle A(\mu) u, M(\varepsilon ; \lambda) u\rangle-\left\langle M(\varepsilon ; \lambda) u, A(\mu)^{*} u\right\rangle \\
=\left\langle A(\mu) f_{\delta} u, B(\varepsilon ; \lambda) f_{\delta} u\right\rangle-\left\langle B(\varepsilon ; \lambda) f_{\delta} u, A(\mu)^{*} f_{\delta} u\right\rangle \\
\quad+\left\langle\left[f_{\delta}, A(\mu)\right] u, B(\varepsilon ; \lambda) f_{\delta} u\right\rangle+\left\langle B(\varepsilon ; \lambda) f_{\delta} u,\left[A(\mu)^{*}, f_{\delta}\right] u\right\rangle
\end{aligned}
$$

(3.2) implies that

$$
\begin{aligned}
& {[M(\varepsilon ; \lambda), A(\mu)]} \\
& =f_{\delta}[B(\varepsilon ; \lambda), A(\mu)] f_{\delta}+f_{\delta} B(\varepsilon ; \lambda)\left[f_{\delta}, A(\mu)\right]+\left[f_{\delta}, A(\mu)\right] B(\varepsilon ; \lambda) f_{\delta} .
\end{aligned}
$$


For $u \in H^{2}\left(\boldsymbol{R}^{n}\right) \cap D(A)$ satisfing $A u \in H^{1}\left(\boldsymbol{R}^{n}\right)$, we define the commutator $[B(\varepsilon ; \lambda), A]$ as follows

$$
\begin{aligned}
\langle i[B(\varepsilon ; \lambda), A] u, u\rangle & =\sum_{j=1}^{4}\left\langle i\left[B_{j}(\varepsilon ; \lambda), A\right] u, u\right\rangle \\
& =\sum_{j=1}^{4} i\left(\left\langle A u, B_{j}(\varepsilon ; \lambda) u\right\rangle-\left\langle B_{j}(\varepsilon ; \lambda) u, A u\right\rangle\right),
\end{aligned}
$$

where

$$
\begin{aligned}
B_{1}(\varepsilon ; \lambda) & =-2 \Delta+\lambda\left(x \cdot \nabla E_{L, \varepsilon}\right), \\
B_{2}(\varepsilon ; \lambda) & =-\lambda\left(\left(E_{S, \varepsilon} x \cdot \nabla\right) *+E_{S, \varepsilon} x \cdot \nabla+n E_{S, \varepsilon}\right) \\
& =-\lambda\left(\nabla^{*} \cdot x E_{S, \varepsilon}+E_{S, \varepsilon} x \cdot \nabla+n E_{S, \varepsilon}\right), \\
B_{3}(\varepsilon ; \lambda) & =-\lambda T_{\varphi}^{*} V_{\varepsilon}^{r}\left(E_{L}^{+0}-E_{L}^{-0}\right) T_{\varphi}, \\
B_{4}(\varepsilon ; \lambda) & =-\lambda\left(R_{\varphi}^{r}\right) *\left(E_{L}^{+0}-E_{L}^{-0}\right) R_{\varphi}^{r} .
\end{aligned}
$$

Then the $i[B(\varepsilon ; \lambda), A]$ has an extension $[B(\varepsilon ; \lambda), A]^{0}$ to be a bounded operator from $H^{2}\left(\boldsymbol{R}^{n}\right)$ to $H^{-2}\left(\boldsymbol{R}^{n}\right)$ (see Appendix). Let $u \in L^{2}$. Again, noting that $A(\mu) H^{k}\left(\boldsymbol{R}^{n}\right) \subset H^{k}\left(\boldsymbol{R}^{n}\right)(k= \pm 1, \pm 2)$, we have

$$
\begin{aligned}
&\left\langle A(\mu) f_{\delta} u, B(\varepsilon ; \lambda) f_{\delta} u\right\rangle-\left\langle B(\varepsilon ; \lambda) f_{\delta} u, A(\mu)^{*} f_{\delta} u\right\rangle \\
&=\mu^{2}\left(\left\langle B(\varepsilon ; \lambda)(A+i \mu)^{-1} f_{\delta} u, f_{\delta} u\right\rangle-\left\langle f_{\delta} u, B(\varepsilon ; \lambda)(A-i \mu)^{-1} f_{\delta} u\right\rangle\right) \\
&=\mu^{2}\left(\left\langle B(\varepsilon ; \lambda)(A+i \mu)^{-1} f_{\delta} u, A(A-i \mu)^{-1} f_{\delta} u\right\rangle\right. \\
&\left.-\left\langle A(A+i \mu)^{-1} f_{\delta} u, B(\varepsilon ; \lambda)(A-i \mu)^{-1} f_{\delta} u\right\rangle\right) .
\end{aligned}
$$

(3.4) implies that

$$
f_{\delta}[B(\varepsilon ; \lambda), A(\mu)] f_{\delta}=f_{\delta} i \mu(A+i \mu)^{-1}[B(\varepsilon ; \lambda), A]^{0} i \mu(A+i \mu)^{-1} f_{\delta}
$$

as an operator on $L^{2}$. (3.1) and (3.5) imply that

$$
s-\lim _{|\mu| \rightarrow \infty} f_{\delta}[B(\varepsilon ; \lambda), A(\mu)] f_{\delta}=f_{\delta}[B(\varepsilon ; \lambda), A]^{0} f_{\delta},
$$

for all $\lambda \in\left(\lambda_{0}-\delta, \lambda_{0}+\delta\right)$, as an operator on $L^{2}$. Using Lemma 3.1, by the same way as in the proof of Lemma 2.4 of Weder [13], we can prove that

$$
\begin{gathered}
s-\lim _{|\mu| \rightarrow \infty}(-\Delta+1)^{1 / 2}\left[f_{\delta}, A(\mu)\right](-\Delta+1)^{1 / 2} \\
=(-\Delta+1)^{1 / 2}\left[f_{\delta}, A\right]^{0}(-\Delta+1)^{1 / 2}
\end{gathered}
$$

for all $\lambda \in\left(\lambda_{0}-\delta, \lambda_{0}+\delta\right)$, as an operator on $L^{2}$. Hence by (3.2), (3.6) and (3.7), we have

$$
\begin{aligned}
& s-\lim _{|\mu| \rightarrow \infty}[M(\varepsilon ; \lambda), A(\mu)] \\
& \quad=f_{\delta}[B(\varepsilon ; \lambda), A]^{0} f_{\delta}+f_{\delta} B(\varepsilon ; \lambda)\left[f_{\delta}, A\right]^{0}+\left[f_{\delta}, A\right]^{0} B(\varepsilon ; \lambda) f_{\delta}
\end{aligned}
$$


for all $\lambda \in\left(\lambda_{0}-\delta, \lambda_{0}+\delta\right)$, as an operator on $L^{2}$. We define $[M(\varepsilon ; \lambda), A]^{0}$ by the right side of (3.8).

Finally, for $u, v \in D(A)$, we have

$$
\begin{aligned}
\left\langle[M(\varepsilon ; \lambda), A]^{0} u, v\right\rangle & =\lim _{|\mu| \rightarrow \infty}(\langle A(\mu) u, M(\varepsilon ; \lambda) v\rangle-\langle M(\varepsilon ; \lambda) u, A(\mu) v\rangle) \\
& =\langle A u, M(\varepsilon ; \lambda) v\rangle-\langle M(\varepsilon ; \lambda) u, A v\rangle .
\end{aligned}
$$

We can prove the following lemma by a straightforward calculation.

LEMMA 3.4. Let $\lambda_{0}-\delta<\lambda<\lambda_{0}+\delta, 0<\varepsilon<1$. There exists a positive constant $C$ independent of $\lambda$ and $\varepsilon$ such that

$$
\begin{aligned}
& \left\|(-\Delta+1)^{-1 / 2}(B(\lambda)-B(\varepsilon ; \lambda))(-\Delta+1)^{-1 / 2}\right\| \leqq C \varepsilon^{\theta}, \\
& \left\|(-\Delta+1)^{-1 / 2}(d / d \varepsilon) B(\varepsilon ; \lambda)(-\Delta+1)^{-1 / 2}\right\| \leqq C \varepsilon^{0-1}, \\
& \left\|(-\Delta+1)^{-1}[B(\varepsilon ; \lambda), A]^{0}(-\Delta+1)^{-1}\right\| \leqq C \varepsilon^{\theta-1},
\end{aligned}
$$

where $B(\lambda)=[H(\lambda), A]^{0}$.

Let $\lambda_{0}>0$. By Theorem 1.1(i), for the compact operator $K\left(\lambda_{0}\right)$ on $L^{2}$, we have

$$
\left\|f_{\delta}\left(H\left(\lambda_{0}\right)\right) K\left(\lambda_{0}\right) f_{\delta}\left(H\left(\lambda_{0}\right)\right)\right\|=o(1)(\delta \longrightarrow 0) .
$$

We can also show that

$$
\left\|f_{\delta}(H(\lambda))-f_{\delta}\left(H\left(\lambda_{0}\right)\right)\right\| \leqq C \delta,
$$

where $C>0$ depends on only $\lambda_{0}$. Thus by Lemma 2.4 , we can take $\delta$ so small that

$$
\begin{aligned}
M(\lambda) & \equiv f_{\delta}(H(\lambda)) i[H(\lambda), A]^{0} f_{\delta}(H(\lambda)) \\
& \geqq(\alpha / 2) f_{\delta}(H(\lambda))^{2}
\end{aligned}
$$

in the form sense.

Moreover, (3.9) together with Lemma 3.4(i) implies that

$$
M(\varepsilon ; \lambda) \geqq \gamma f_{\delta}(H(\lambda))^{2}
$$

for $\varepsilon>0$ small enough, where $\gamma>0$.

It follows from $(3.10)$ that $M(\varepsilon ; \lambda)$ is non-negative and hence we define an operator, $G_{\kappa}(\varepsilon ; \lambda)$, on $L^{2}$ by

$$
G_{\kappa}(\varepsilon ; \lambda)=(H(\lambda)-\lambda-i \kappa E(x)-i \varepsilon M(\varepsilon ; \lambda))^{-1}
$$

for $0<\kappa<1$ and $0<\varepsilon \ll 1$. 
LEMMA 3.5. Let $0<\kappa<1, \lambda_{0}-\delta<\lambda<\lambda_{0}+\delta$ and $0<\varepsilon \ll 1$. There exists $a$ positive constant $C$ independent of $\kappa, \lambda$ and $\varepsilon$ such that

$$
\left\|G_{\kappa}(\varepsilon ; \lambda)\right\| \leqq C \varepsilon^{-1} .
$$

For a proof of Lemma 3.5, see that of Lemma 5.3 of Kikuchi and Tamura [8] or Lemma 3.2 of Tamura [11].

For $1 / 2<\alpha \leqq 1$, we write

where

$$
F_{\kappa}(\varepsilon ; \lambda)=A_{\alpha}(\varepsilon) G_{\kappa}(\varepsilon ; \lambda) A_{\alpha}(\varepsilon),
$$

$$
A_{\alpha}(\varepsilon)=(1+|A|)^{-\alpha}(1+\varepsilon|A|)^{\alpha-1} .
$$

Differentiating $F_{\kappa}(\varepsilon ; \lambda)$ in $\varepsilon$, we have

$$
\begin{aligned}
(d / d \varepsilon) F_{\kappa}(\varepsilon ; \lambda)= & \left((d / d \varepsilon) A_{\alpha}(\varepsilon)\right) G_{\kappa} A_{\alpha}(\varepsilon)-i A_{\alpha}(\varepsilon) G_{\kappa} M(\varepsilon ; \lambda) G_{\kappa} A_{\alpha}(\varepsilon) \\
& -i \varepsilon A_{\alpha}(\varepsilon) G_{\kappa}((d / d \varepsilon) M(\varepsilon ; \lambda)) G_{\kappa} A_{\alpha}(\varepsilon) \\
& +A_{\alpha}(\varepsilon) G_{\kappa}\left((d / d \varepsilon) A_{\kappa}(\varepsilon)\right) .
\end{aligned}
$$

Repeating the argument in the proof of Lemma 2.9 of Weder [13], we can show from Lemma 3.2 and 3.3

$$
G_{\kappa}(\varepsilon ; \lambda) D(A) \subset D(A) \cap H^{2}\left(\boldsymbol{R}^{n}\right) .
$$

Let $g_{\delta}(p)=1-f_{\delta}(p)$. We write in brief $f_{\delta}$ and $g_{\delta}$ for $f_{\delta}(H(\lambda))$ and $g_{\delta}(H(\lambda))$ respectively.

Using (3.11), (3.12) and Lemma 3.3, we can decompose $(d / d \varepsilon) F_{\kappa}(\varepsilon ; \lambda)$ as a form on $L^{2}$

$$
(d / d \varepsilon) F_{\kappa}(\varepsilon ; \lambda)=\sum_{j=1}^{9} Y_{k}^{j}(\varepsilon ; \lambda),
$$

where

$$
\begin{aligned}
& Y_{\kappa}^{1}(\varepsilon ; \lambda)=i A_{\alpha}(\varepsilon) G_{\kappa} f_{\delta} B(\varepsilon ; \lambda) g_{\delta} G_{\kappa} A_{\alpha}(\varepsilon), \\
& Y_{\kappa}^{2}(\varepsilon ; \lambda)=i A_{\alpha}(\varepsilon) G_{\kappa} g_{\delta} B(\varepsilon ; \lambda) f_{\delta} G_{\kappa} A_{\alpha}(\varepsilon), \\
& Y_{\kappa}^{3}(\varepsilon ; \lambda)=i A_{\alpha}(\varepsilon) G_{\kappa} g_{\delta} B(\varepsilon ; \lambda) g_{\delta} G_{\kappa} A_{\alpha}(\varepsilon), \\
& Y_{\kappa}^{4}(\varepsilon ; \lambda)=-i A_{\alpha}(\varepsilon) G_{\kappa}(B(\varepsilon ; \lambda)-B(\lambda)) G_{\kappa} A_{\alpha}(\varepsilon) \\
& Y_{\kappa}^{5}(\varepsilon ; \lambda)=-i A_{\alpha}(\varepsilon) G_{\kappa}[H(\lambda)-\lambda-i \kappa E(x)-i \varepsilon M(\varepsilon ; \lambda), A] G_{\kappa} A_{\alpha}(\varepsilon), \\
& Y_{\kappa}^{6}(\varepsilon ; \lambda)=\kappa A_{\alpha}(\varepsilon) G_{\kappa}[E(x), A] G_{\kappa} A_{\alpha}(\varepsilon), \\
& Y_{\kappa}^{7}(\varepsilon ; \lambda)=\varepsilon A_{\alpha}(\varepsilon) G_{\kappa}[M(\varepsilon ; \lambda), A] G_{\kappa} A_{\alpha}(\varepsilon), \\
& Y_{\kappa}^{8}(\varepsilon ; \lambda)=-i \varepsilon A_{\alpha}(\varepsilon) G_{\kappa}((d / d \varepsilon) M(\varepsilon ; \lambda)) G_{\kappa} A_{\alpha}(\varepsilon), \\
& Y_{\kappa}^{r}(\varepsilon ; \lambda)=\left((d / d \varepsilon) A_{\alpha}(\varepsilon)\right) G_{\kappa} A_{\kappa}(\varepsilon)+A_{\alpha}(\varepsilon) G_{\kappa}\left((d / d \varepsilon) A_{\alpha}(\varepsilon)\right) .
\end{aligned}
$$


We need the following lemmas to estimate each term of right side of (3.13).

LEMmA 3.6. Let $0<\kappa<1, \lambda_{0}-\delta<\lambda<\lambda_{0}+\delta$, and $0<\varepsilon \ll 1$. There exists $a$ positive constant independent of $\kappa, \lambda$ and $\varepsilon$ such that

$$
\begin{aligned}
& \left\|(-\Delta+1)^{1 / 2} f_{\delta} G_{\kappa}(\varepsilon ; \lambda) A_{\alpha}(\varepsilon)\right\| \leqq C \varepsilon^{-1 / 2}\left\|F_{\kappa}\right\|^{1 / 2}, \\
& \left\|(-\Delta+1)^{1 / 2} g_{\delta} G_{\kappa}(\varepsilon ; \lambda) A_{\alpha}(\varepsilon)\right\| \leqq C, \\
& \left\|(-\Delta+1)^{1 / 2} G_{\kappa}(\varepsilon ; \lambda) A_{\alpha}(\varepsilon)\right\| \leqq C \kappa^{-1 / 2}\left\|F_{\kappa}\right\|^{1 / 2} .
\end{aligned}
$$

For a proof of Lemma 3.6, see that of Lemma 5.5 of Kikuchi and Tamura [8].

LEMmA 3.7. Let $\lambda_{0}-\delta<\lambda<\lambda_{0}+\delta$ and $0<\varepsilon \ll 1$. There exists a positive constant independent of $\lambda$ and $\varepsilon$ such that

$$
\left\|[M(\varepsilon ; \lambda), A]^{0}\right\| \leqq C \varepsilon^{\theta-1} .
$$

Proof. By the definition of $[M(\varepsilon ; \lambda), A]^{0}$, we estimate each term of right side of (3.8).

Lemma 3.4 (iii) implies that

$$
\left\|f_{\delta}[B(\varepsilon ; \lambda), A]^{0} f_{\delta}\right\| \leqq C \varepsilon^{\theta-1} .
$$

Noting the definition of $B(\varepsilon ; \lambda)$ and $(3.7)$, we also have that

$$
\left\|f_{\delta} B(\varepsilon ; \lambda)\left[f_{\delta}, A\right]^{0}\right\|,\left\|\left[f_{\delta}, A\right]^{0} B(\varepsilon ; \lambda) f_{\delta}\right\| \leqq C,
$$

where $C>0$ is independent of $\varepsilon$. Thus, the proof of Lemma 3.7 is now complete.

Using lemma 3.6 and 3.7 , we can evaluate the norm of $Y^{\prime}{ }_{k}^{j}, 1 \leqq j \leqq 9$, and obtain the following differential inequality (see Tamura [12])

$$
\left\|(d / d \varepsilon) F_{\kappa}(\varepsilon ; \lambda)\right\| \leqq C\left(\varepsilon^{\alpha-1}+\varepsilon^{\alpha-3 / 2}\left\|F_{\kappa}\right\|^{1 / 2}+\varepsilon^{\theta-1}\left\|F_{\kappa}\right\|\right) .
$$

Let $\varepsilon_{0}, 0<\varepsilon_{0} \ll 1$. Then by Lemma 3.5, we have

$$
\left\|F_{\kappa}\left(\varepsilon_{0} ; \lambda\right)\right\| \leqq C \varepsilon_{0}^{-1} .
$$

By (3.10) and (3.11), we immediately obtain

$$
\left\|F_{\kappa}(\varepsilon ; \lambda)\right\| \leqq C,
$$

where $C>0$ independent of $0<\kappa<1,0<\varepsilon<\varepsilon_{0}$ and $\lambda_{0}-\delta<\lambda<\lambda_{0}+\delta$.

In order to prove (ii) and (iii) of theorem 1.1, we need the following lemma (see Weder [13]).

LEMMA 3.8. Let $\lambda_{0}-\delta<\lambda<\lambda_{0}+\delta$ and $0<\varepsilon \ll 1$. There exists a positive constant 
$C$ independent of $\lambda$ and $\varepsilon$ such that

where

$$
\left\|A_{\alpha}(\varepsilon)^{-1} f_{\delta} X_{\alpha}(\varepsilon)\right\| \leqq C
$$

$$
X_{\alpha}(\varepsilon)=\left(1+|x|^{2}\right)^{-\alpha / 2}\left(1+\varepsilon^{2}|x|^{2}\right)^{(\alpha-1) / 2} .
$$

Proof. By the same argument as in Weder [13], we can show that $(1+\varepsilon|A|) f_{\delta}\left(1+\varepsilon^{2}|x|^{2}\right)^{-1 / 2}$ is a bounded operator on $L^{2}$ and

$$
\left\|(1+\varepsilon|A|) f_{\delta}\left(1+\varepsilon^{2}|x|^{2}\right)^{-1 / 2}\right\| \leqq C,
$$

where $C$ indepedent of $\varepsilon$ and $\lambda\left(0<\varepsilon \ll 1, \lambda_{0}-\delta<\lambda<\lambda_{0}+\delta\right)$.

Take $\varepsilon=1$, then we also have

$$
\left\|(1+|A|) f_{\delta}\left(1+|x|^{2}\right)^{-1 / 2}\right\| \leqq C
$$

we obtain

$$
\left\|A_{\alpha}(\varepsilon)^{-1} f_{\delta} X_{\alpha}(\varepsilon)\right\| \leqq C
$$

by interpolation between (3.17) and (3.18).

Proof of Theorem 1.1(II), (III): Using (3.1) and Lemma 3.8, we have

$$
\left\|X_{\alpha}(\varepsilon) f_{\delta} G_{\kappa}(\varepsilon ; \lambda) f_{\delta} X_{\alpha}(\varepsilon)\right\| \leqq C .
$$

Moreover, since

we have

$$
\left\|g_{\delta} G_{\kappa}(\varepsilon ; \lambda)\right\|,\left\|G_{\kappa}(\varepsilon ; \lambda) g_{\delta}\right\| \leqq C,
$$

$$
\begin{aligned}
& \left\|X_{\alpha}(\varepsilon) g_{\delta} G_{\kappa}(\varepsilon ; \lambda) f_{\delta} X_{\alpha}(\varepsilon)\right\| \leqq C, \\
& \left\|X_{\alpha} G_{\kappa}(\varepsilon ; \lambda) g_{\delta} X_{\alpha}(\varepsilon)\right\| \leqq C .
\end{aligned}
$$

By (3.19), (3.20) and (3.21), we obtain

$$
\left\|X_{\alpha}(\varepsilon) G(\varepsilon ; \lambda) X_{\alpha}(\varepsilon)\right\| \leqq C .
$$

We write

$$
F_{\kappa}(\varepsilon ; \lambda)=X_{\alpha}(\varepsilon) G_{\kappa}(\varepsilon ; \lambda) X_{\alpha}(\varepsilon) .
$$

We can prove (3.14) for $F_{\kappa}(\varepsilon ; \lambda)$ defined above. Thus using (3.22), we have

$$
\left\|(d / d \varepsilon) F_{\kappa}(\varepsilon ; \lambda)\right\| \leqq C \varepsilon^{\beta-1}
$$

where $0<\beta<1$.

Finally, by (3.22) and (3.23), we obtain Theorem 1.1(ii), (iii).

\section{Appendix.}

In this section we show that the commutator $i\left[B_{j}(\varepsilon ; \lambda), A\right](j=1,2,3,4)$ is 
extended to a bounded operator from $H^{2}\left(\boldsymbol{R}^{n}\right)$ to $H^{-2}\left(\boldsymbol{R}^{n}\right)$. We write $i\left[B_{j}(\varepsilon ; \lambda)\right.$, $A]^{0}(j=1,2,3,4)$ for the extended operators.

We define an operator $P_{\varphi}^{r}$ as

$$
P_{\varphi}^{r} u=\chi_{|y| \leq r}(y)\left(\left(y \cdot \nabla_{y} \varphi(y)-\varphi(y)\right) \varphi(y)\right)^{1 / 2} T_{\varphi} u,
$$

for $u \in \boldsymbol{S}\left(\boldsymbol{R}^{n}\right)$.

LEMMA 4.1. $\quad P_{\varphi}^{r}$ is extended to a bounded operator from $H^{1}\left(\boldsymbol{R}^{n}\right)$ to $L^{2}\left(\boldsymbol{R}^{n-1}\right)$.

PROoF. If $0<\sigma<1 / 2$, there exist some $s^{\prime}>1 / 2$ and $p^{\prime}>n-1$ such that $n-1-2 \sigma p^{\prime}>0$ and $(n-1) / 2 p^{\prime}+s^{\prime}=1$. Then we can prove that

$$
\left|P_{\varphi}^{r} u\right|_{L 2\left(R^{n-1}\right)} \leqq C r^{\left(n-1-2 \sigma p^{\prime}\right) / 2 p^{\prime}}|u|_{H^{2(R}\left(R^{n}\right)}
$$

in the same way as in the proof of (2.14).

By a straightforward calculation, the form $i\left[B_{j}(\varepsilon ; \lambda), A\right](j=1,2)$ is extended to a bounded operator from $H^{2}\left(\boldsymbol{R}^{n}\right)$ to $H^{-2}\left(\boldsymbol{R}^{n}\right)$. Moreover, we have that

$$
\begin{aligned}
i\left[B_{1}(\varepsilon ; \lambda), A\right]^{0}= & -4 \Delta+\lambda\left(\left(x \cdot \nabla E_{L, \varepsilon}\right)(x \cdot \nabla)+\left(\nabla^{*} \cdot x\right)\left(x \cdot \nabla E_{L, \varepsilon}\right)+n\left(x \cdot \nabla E_{L, \varepsilon}\right)\right), \\
i\left[B_{2}(\varepsilon ; \lambda), A\right]^{0}= & -\lambda\left(E_{S, \varepsilon}(x \cdot \nabla)(x \cdot \nabla)+\left(E_{S, \varepsilon}(x \cdot \nabla)(x \cdot \nabla)\right)^{*}+2(\nabla * \cdot x) E_{S, \varepsilon}(x \cdot \nabla)\right. \\
& \left.+2 n E_{S, \varepsilon}(x \cdot \nabla)+2 n\left(\nabla^{*} \cdot x\right) E_{S, \varepsilon}+n^{2} E_{S, \varepsilon}\right) .
\end{aligned}
$$

Let $u \in H^{2}\left(\boldsymbol{R}^{n}\right) \cap D(A)$ satisfying $A u \in H^{1}\left(\boldsymbol{R}^{n}\right)$. Then we have that

$$
\begin{aligned}
\left\langle i\left[B_{3}(\varepsilon ; \lambda), A\right] u, u\right\rangle= & -\lambda\left(\left\langle y \cdot T_{\varphi} \nabla_{y} u, V_{\varepsilon}^{r}\left(E_{L}^{+0}-E_{L}^{-0}\right) T_{\varphi} u\right\rangle_{L^{2}\left(R^{n-1}\right)}\right. \\
& +\left\langle\varphi(y) T_{\varphi} \partial_{z} u, V_{\varepsilon}^{r}\left(E_{L}^{+0}-E_{L}^{-0}\right) T_{\varphi} u\right\rangle_{L^{2}\left(R^{n-1}\right)} \\
& +\left\langle V_{\varepsilon}^{r}\left(E_{L}^{+0}-E_{L}^{-0}\right) T_{\varphi} u, y \cdot T_{\varphi} \nabla_{y} u\right\rangle_{L^{2}\left(R^{n-1}\right)} \\
& +\left\langle V_{\varepsilon}^{r}\left(E_{L}^{+0}-E_{L}^{-0}\right) T_{\varphi} u, \varphi(y) T_{\varphi} \partial_{z} u\right\rangle_{L^{2}\left(R^{n-1}\right)} \\
& \left.+n\left\langle V_{\varepsilon}^{r}\left(E_{L}^{+0}-E_{L}^{-0}\right) T_{\varphi} u, T_{\varphi} u\right\rangle_{L^{2}\left(R^{n-1}\right)}\right), \\
\left\langle i\left[B_{4}(\varepsilon ; \lambda), A\right] u ' u\right\rangle= & -\lambda\left(\left\langle y \cdot R_{\varphi}^{r} \nabla_{y} u,\left(E_{L}^{+0}-E_{L}^{-0}\right) R_{\varphi}^{r} u\right\rangle_{L^{2}\left(R^{n-1}\right)}\right. \\
& +\left\langle P_{\varphi}^{r} \partial_{z} u,\left(E_{L}^{+0}-E_{L}^{-0}\right) P_{\varphi}^{r} u\right\rangle_{L^{2}\left(R^{n-1}\right)} \\
& +\left\langle\left(E_{L}^{+0}-E_{L}^{-0}\right) R_{\varphi}^{r} u, y \cdot R_{\varphi}^{r} \nabla_{y} u\right\rangle_{L^{2}\left(R^{n-1}\right)} \\
& +\left\langle\left(E_{L}^{+0}-E_{L}^{-0}\right) P_{\varphi}^{r} u, P_{\varphi}^{r} \partial_{z} u\right\rangle_{L^{2}\left(R^{n-1}\right)} \\
& \left.+n\left\langle\left(E_{L}^{+0}-E_{L}^{-0}\right) R_{\varphi}^{r} u, R_{\varphi}^{r} u\right\rangle_{L^{2}\left(R^{n-1}\right)}\right) .
\end{aligned}
$$

Lemma 2.1 and (4.1) imply that $i\left[B_{3}(\varepsilon ; \lambda), A\right]$ is extended to a bounded operator from $H^{2}\left(\boldsymbol{R}^{n}\right)$ to $H^{-2}\left(\boldsymbol{R}^{n}\right)$. Lemma 4.1, (2.14) and (4.2) imply that 
$i\left[B_{4}(\varepsilon ; \lambda), A\right]$ is extended to a bounded operator from $H^{2}\left(\boldsymbol{R}^{n}\right)$ to $H^{-2}\left(\boldsymbol{R}^{n}\right)$. We also have that

$$
\begin{aligned}
i\left[B_{3}(\varepsilon ; \lambda), A\right]^{0}= & -\lambda\left(T_{\varphi}^{*}\left(E_{L}^{+0}-E_{L}^{-0}\right) V_{\varepsilon}^{r} y \cdot T_{\varphi} \nabla_{y}+\left(T_{\varphi} \nabla_{y}\right) * \cdot y\left(E_{L}^{+0}-E_{L}^{-0}\right) V_{\varepsilon}^{r} T_{\varphi}\right. \\
& +T_{\varphi}^{*}\left(E_{L}^{+0}-E_{L}^{-0}\right) \varphi(y) V_{\varepsilon}^{r} T_{\varphi} \partial_{z}+\left(T_{\varphi} \partial_{z}\right) *\left(E_{L}^{+0}-E_{L}^{-0}\right) \varphi(y) V_{\varepsilon}^{r} T_{\varphi} \\
& \left.+n T_{\varphi}^{*}\left(E_{L}^{+0}-E_{L}^{-0}\right) V_{\varepsilon}^{r} T_{\varphi}\right), \\
i\left[B_{4}(\varepsilon ; \lambda), A\right]^{0}= & -\lambda\left(\left(R_{\varphi}^{r}\right)^{*}\left(E_{L}^{+0}-E_{L}^{-0}\right) y \cdot R_{\varphi}^{r} \nabla_{y}+\left(R_{\varphi}^{r} \nabla_{y}\right) * \cdot y\left(E_{L}^{+0}-E_{L}^{-0}\right) R_{\varphi}^{r}\right. \\
& +\left(P_{\varphi}^{r}\right)^{*}\left(E_{L}^{+0}-E_{L}^{-0}\right) P_{\varphi}^{r} \partial_{z}+\left(P_{\varphi}^{r} \partial_{z}\right) *\left(E_{L}^{+0}-E_{L}^{-0}\right) P_{\varphi}^{r} \\
& \left.+n\left(R_{\varphi}^{r}\right) *\left(E_{L}^{+0}-E_{L}^{-0}\right) R_{\varphi}^{r}\right) .
\end{aligned}
$$

We define an operator $i[B(\varepsilon ; \lambda), A]^{0}$ as

$$
i[B(\varepsilon ; \lambda), A]^{0}=\sum_{j=1}^{4} i\left[B_{j}(\varepsilon ; \lambda), A\right]^{0} .
$$

Thus, the form $i[B(\varepsilon ; \lambda), A]$ is extended to a bounded operator from $H^{2}\left(\boldsymbol{R}^{n}\right)$ to $H^{-2}\left(\boldsymbol{R}^{n}\right)$.

REMARK. We can consider that $i\left[B_{1}(\varepsilon ; \lambda) ; A\right]^{0}$ belongs to $\mathfrak{B}\left(H^{1}, H^{-1}\right)$ and $i\left[B_{3}(\varepsilon ; \lambda), A\right]^{0}$ belongs to $\mathfrak{B}\left(H^{1+s}, H^{-1-s}\right)$, where $s>1 / 2$.

\section{References}

[1] Ben-Artzi. M, Dermenjian. Y and Guillot. J.C, Acoustic waves in perturbed stratified fluids: a spectral theory, Comm. Partial Differential Equations 14 (1989), 479-517.

[2] Debièvre. S and Pravica. W, Spectral analysis for optical fibres and stratified fluids I: the limiting absorption principle, J. Func. Anal 98 (1991), 404-436.

[3] Dermenjian. Y and Guillot. J.C, Theéorie spectrale de la propagation des ondes acoustiques dans un milieu stratifié perturbé, J. Defferential Equations 62 (1986), 357-409.

[4] Eastham. M.S and Kalf. H, "Schrödinger-type operators with continuous spectra, Resaech note in Math", Pitman, 1982.

[5] Eidus. D, The limiting absorption and amplitude principle for the diffraction problem with two unbounded media, Comm. Math. Phys 107 (1986), 27-38.

[6] Froese. $R$ and Herbst. I, Exponential bounds and absence of positive eigenvalues for $N$-body Schrödinger operator, Comm. Math. Phys 87 (1982), 429-447.

[7] Iwashita. H, Spectral theory for symmetric systems in an exterior domain, Tsukuba J. Math 11 (1987), 241-256.

[8] Kikuchi. $\mathrm{K}$ and Tamura. $\mathrm{H}$, Limiting amplitude principle for acoustic propagators in perturbed stratified fluids, J. Defferential Equations 93 (1991), 260-282.

[9] Mourre. E, Absence of singular continuous spectrum for certain self-adjoint operators, Comm. Math. Phys 78 (1981), 391-408.

[10] Perry. P, Sigal. I. M and Simon. B, Spectral analysis of $N$-body Schrödinger operators, Ann of Math 114 (1981), 519-567. 
[11] Tamura. H, Resolvent estimates at low frequencies and limiting ampliting amplitude principle for acoustic propagators, J. Math. Soc. Japan 41 (1989), 549-575.

[12] - Principle of limiting absorption for $N$-body Schrödinger operators, A remark on the commutator method, Lett. in Math. Phys 17 (1989), 31-36.

[13] Weder. R, Spectral analysis of strongly propagator systems, J. Reine Angew. Math. 354 (1984), 95-122.

[14] - Spectral and scattering theory in perturbed stratified fluids, J. Math. Pures et Appl 64 (1985), 149-173.

[15] - Spectral and scattering theory in perturbed stratified fluids II, transmission and exterior domains, J. Defferential Equations 64 (1986), 109-131.

[16] - The limiting absorption principle at thresholds, J. Math. pures at Appl 67 (1988), 318-338.

[17] Wilcox. C. H, Spectral analysis of the Pekeris operator in the theory of acoustic wave propagation in Shallow water, Arch. Rational Mech. Anal 60 (1976), 259-300.

University of Tsukuba, Institute of Mathematics, Ibaraki, 305, Japan 